\title{
Vibrotactile masking: Some spatial and temporal aspects'
}

\author{
RICHARD D. GILSON ${ }^{2}$ \\ PRINCETON UNIVERSITY
}

Threshold elevations (TE) were determined for three Os at a test site on the left thigh with a masker placed singly at each of 10 widespread loci on the trunk. Although lateral changes in masker placement did not produce a significant alteration in $T E(p>.10)$, there was a systematic decrease in $T E$ with longitudinal separation between masker and test sites on the body. Appropriate manipulations of the time interval between masker and test signals were found effective in offsetting these decreases. It appeared that longitudinal separations were differentially affecting temporal delays by producing unequal neural distances from the test and masker sites to the CNS. There were, however, some spatial changes that could not be accounted for by temporal offsets. Thresholds at a finger test site were significantly less elevated $(p<.01)$ by contralateral placement of maskers than by ipsilateral placement, and the particular test site chosen for threshold determinations, either index finger or thigh, determined the amount of TE.

The few studies that have dealt with problems of intrasensory interaction on the skin with the standard masking procedure have confined stimulation to a relatively circumscribed area of the body, i.e., the fingers, hands, and forearms (Halliday \& Mingay, 1961; Rosner, 1961; Schmid, 1961; Sherrick, 1960, 1964; Uttal, 1960). There was good reason for this not only because of high sensitivity and ease of access, but also since most cutaneous studies where sensory interaction might be of concern have similarly used these areas.

Recently, however, there have been a growing number of studies employing more extensive areas of the body (Alluisi, Morgan, \& Hawkes, 1965; Craig, 1966; Geldard, 1966; Geldard $\&$ Sherrick, 1965). Although the orientations of these studies are varied, they do generate questions as to what sorts of interactions are taking place over widespread bodily areas.

The present investigation was undertaken to assay with the masking procedure some of the spatial and temporal properties of the somesthetic system over extensive areas of the human integument.

\section{EXPERIMENT 1}

Simple spatial variations were employed at various widespread bodily loci, in order to assess the limits of spatial interaction.

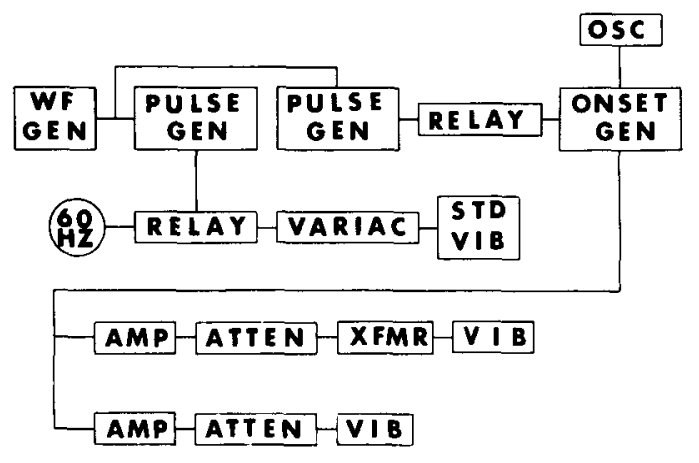

Fig. 1. A block diagram of the apparatus.

\section{Apparatus}

The apparatus was designed to provide one vibrator with a signal controlled for proper frequency, intensity, and duration, with or without the simultaneous presentation of a duplicate signal to another vibrator. A block diagram is shown in Fig. 1. A Hewlett-Packard Model 200 AB audio oscillator supplied the initial signal, which was gated by an onset generator to eliminate switching transients in the vibratory signal. The signal from the onset generator was led in parallel to a Langevin Model $128 \mathrm{XJ}$ amplifier, and to a Bogen Model MO-100, 100-W power amplifier. The former served to amplify the test signal while the latter acted to amplify the masking signal. The test signal was attenuated by a Hewlett-Packard Model 350C attenuator and then led to an impedancematching transformer, a United Transformer Corporation CVL-1, that dropped the impedance from 500 to $50 \mathrm{ohms}$. The masking signal was controlled in amplitude at the output by a Mallory MGT 50 attenuator. No transformer was necessary for the masking signal since a 50-ohm impedance tap of the amplifier matched the impedance of the attenuator and vibrator. The $100-\mathrm{W}$ amplifier was capable of producing the large signal powers required for loudness matches at the body masking sites.

Both the masking and text signals were led to inertia-type vibrators (Sherrick, 1965). The test vibrator was fitted with a rectangular Lucite contactor $3.0 \mathrm{~cm} \times 3.7 \mathrm{~cm}$ and fastened to the test site, the ventral surface of the left thigh, by gluing with collodion. The masking vibrator was secured in place by a Velcro strap. The skin contactor for the masker was a Lucite button $2 \mathrm{~cm}$ in diam.

The onset generator was gated by a relay that was energized by a Tektronix Type 161 pulse generator. By varying the width of the square wave output from the pulse generator, the duration of the vibratory signal could be controlled. Furthermore, onset and offset times of the signal could be manipulated by the appropriate controls in the onset generator. The pulse generator was triggered at a fixed repetition rate by à Type 162 Tektronix waveform generator. A second pulse generator, triggered by the same waveform generator at a temporal delay, energized a second relay that gated the input of $60-\mathrm{Hz}$ line voltage to a Superior Electric Type $10 \mathrm{~B}$ Powerstat. This signal energized a vibrator to be used for setting of a standard magnitude.

\section{Procedure}

The body sites chosen for stimulation are depicted in Fig. 2. The ventral surface of the left thigh was chosen as a test site since it provided a suitable surface for gluing (horizontal and flat when the $O$ was seated) and it is a relatively large, homogeneous expanse of tissue. The masker sites were chosen because they sampled loci that encompassed roughly the limits of distance from the test site, and they contained equal numbers of contralateral and ipsilateral sites with respect to the test locus.

The general procedure followed in all the experiments to be described involved three steps: (a) A standard sensation magnitude to be equated to all maskers was determined in the following manner. Absolute threshold at the upper left arm (Site A, Fig. 2) was obtained with a Sherrick vibrator attached 


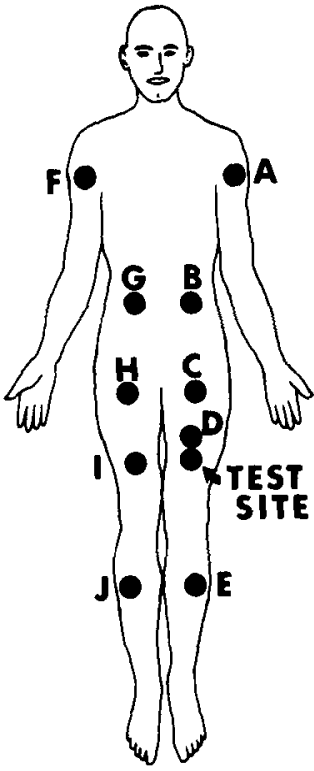
site.

to the arm by a Velcro strap. The signal was a $200-\mathrm{msec}$ burst of $60-\mathrm{Hz}$ vibration. Threshold was taken as the median coil voltage for four ascending trials of a modified method of limits. The coil voltage was then increased $15 \mathrm{~dB}$. (b) The masker was then matched in sensation magnitude to the standard magnitude by the method of average error. The signal of the masker (and the test) was a $200-\mathrm{msec}$ burst of $150-\mathrm{Hz}$ vibration with 20-msec onset and offset times. (c) Masking, as indicated by the threshold elevation (TE) resulting from the presence of a masker, was determined at the test locus by measuring the difference between masked and unmasked thresholds. Each threshold was taken as the median of four ascending and four descending trials in counterbalanced order (abba) of the method of limits. Intensity was changed in 1-dB steps and a $2800-\mathrm{msec}$ blank period was interposed between stimulus bursts during threshold determinations. Threshold elevations were determined for the test site in the presence of a masker placed at each of the masking sites, singly. The choice of masking sites and the order of obtaining masked and unmasked thresholds was changed in a haphazard manner from session to session.

Three Os participated in this experiment as well as in the other experiments to be reported. Each $\mathrm{O}$ had participated in psychophysical experiments prior to the present and was given practice before actual data collection. Two $\mathrm{O}_{\mathrm{s}}$ were males and one $O$ was female. Os were instructed that their criterion for threshold should be the first appearance or disappearance of a vibratory pattern at the test site. At all times during observation sessions, Os received a masking white noise through earphones from a Grason-Stadler Model 455-B noise generator.

\section{Results and Discussion}

Fig. 3 depicts the results for the individual Os and the means for the three Os. The results are plotted as a function of the physical separation along the body's longitudinal axis (head to foot) between the masker and test sites for ipsilateral placement of maskers. In the case of contralateral maskers measurements were made from the site homologous and contralateral to the test site.

There appears to be a systematic variation in the amount of $T E$ as a function of longitudinal separation for both ipsilateral and contralateral placement of maskers. That is, as the longitudinal separation from the test locus or its contralateralhomologous site increased on the body in either direction, the amount of TE decreased. Secondly, a comparison of the effects of ipsilateral and contralateral maskers shows that contralateral maskers were no less effective in elevating the threshold at the test site than ipsilateral maskers, if their respective longitudinal separations were similar $(p>.10$, by the Wilcoxon Signed Rank test).

The finding of contralateral interaction is in agreement with those of others (Békésy, 1955; Bender, 1953; Halliday \& Mingay, 1961; Sherrick, 1964; Yai, 1959). Since with contralateral maskers and even with widely spaced ipsilateral maskers, there is good assurance of physical and peripheral neural isolation from the test site, we may conclude that the interaction is not at the level of the end organs, but at some central site or sites. One further point may be noted. An "isolated" contralateral masker (Site I, Fig. 2) would produce nearly as much TE as a masker situated immediately adjacent to the test site (Site D, Fig. 2) where peripheral interactions, both physical and neural, are presumably having their fullest effects. It therefore would appear that these interactions are probably not nearly as important as central factors in their effects on TE. The latter conclusion serves to.refute the possibility that the decrease in TE with longitudinal separation is caused by a lessening of the effects of peripheral interactions. It would appear that some other as yet unknown factor or factors affect the relation between locus and degree of masking.

\section{EXPERIMENT 2}

It is possible that temporal rather than spatial factors may be effecting the abatement in TE that occurs with longitudinal separation of masker and test loci on the body. Differential time delays between the arrival of the masker and test signals at a central site or sites of interaction, may result from differing neural distances from masker and test loci to the central site. That brief temporal delays between a test and a spatially separate masker affect the amount of $T E$ is a well-documented finding. Sherrick (1964) has shown the effective range for tactual pulse interaction to vary from a 20 -msec delay, with the test preceding the masker, to a peak with simultaneous presentations, to a 20 -msec delay, with the masker preceding the test. Furthermore, that temporal delays of this 20 -msec order can result from spatial separations employed in this study can be shown by calculation from

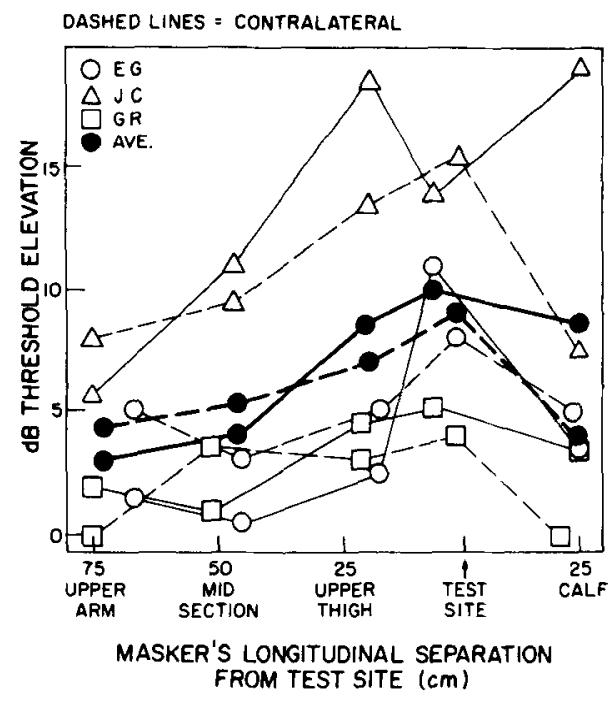

Fig. 3. Threshold elevation at the left thigh test site, as a function of a masker's longitudinal separation (in centimeters) from the test site. 
some past measures of neural signalling speeds in man. An often reported range of neural signalling speeds in sensory nerves of man is from 50 to $70 \mathrm{~m}$ per sec (Golla \& Anatonovitch, 1931; Mayer, 1963). Calculations from these figures for a $75-\mathrm{cm}$ longitudinal separation result in temporal delays of $15.0 \mathrm{msec}$ for $50 \mathrm{~m} / \mathrm{sec}$, and $10.7 \mathrm{msec}$ for $70 \mathrm{~m} / \mathrm{sec}$.

The first experiment in this series was conducted to establish a functional relation for the time interval between the onsets of the masker and test signals, when there was no longitudinal separation between masker and test sites. The purpose was to provide a standard for later comparisons.

The apparatus was modified by the inclusion of a second collateral circuit to permit the generation of two duplicate vibratory signals, previously described, with a specified temporal separation. The output of the audio oscillator went to two onset generators whose outputs in turn went to the two circuits described previously. The onset generators were gated by two relays that were energized by Tektronix Type 161 pulse generators. By varying the delays of the pulse generators it was possible to control precisely the temporal relations between the two signals, i.e., a sequential presentation. The pulse generators were triggered by a single Tektronix waveform generator, Type 162 .

The masker was placed on the right thigh contralateral and homologous to the test site on the left thigh. Seven time intervals of from $-20.0 \mathrm{msec}$ to $+20.0 \mathrm{msec}$ were chosen as intervals between the onset of the masker signal and the onset of the test signal. Negative time intervals are cases where the onset of the test comes before that of the masker.

Multiples of $6.7 \mathrm{msec}$ were used for time delays, since this corresponds to the period of a $150-\mathrm{Hz}$ wave. Using this time delay, in effect, shifts the phase between the two signals by $360 \mathrm{deg}$, or in other words, back into phase. Phase shift between the two signals was therefore controlled.

The results for each $\mathrm{O}$ and the mean are shown in Fig. 4. It is clear from these data that temporal factors do have an effect and that maximum TE appears to occur when there is no time delay between masker and test onsets, or in other words, when the signals occur simultaneously. Furthermore, the data seem roughly symmetrical about the zero time axis, dropping off fairly consistently in TE as temporal delays between the onsets decrease, results which are in agreement with Sherrick's (1964) findings on masking.

Examining the data in light of the differential signal delay hypothesis, it seems plausible if signalling speeds are of the order of 50 to $60 \mathrm{~m} / \mathrm{sec}$, that the drop in TE from 8.0 to $4.3 \mathrm{~dB}$ over a range from 0.0 to $+13.3 \mathrm{msec}$ (masker preceding

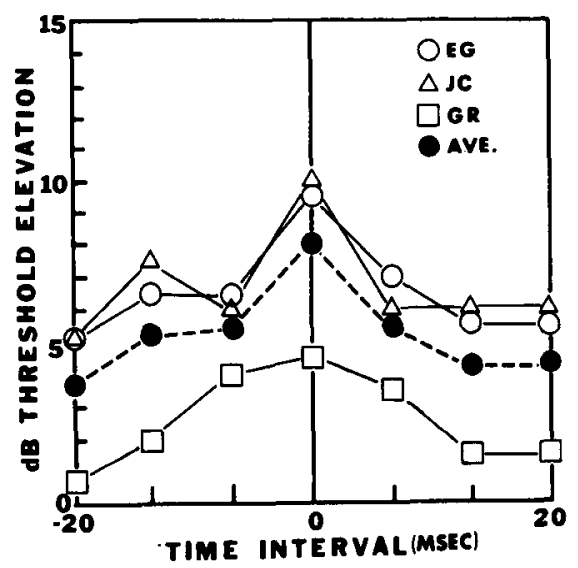

Fig. 4. Threshold elevation as a function of the time interval between masker and test signal onsets. Test site on the lower left thigh; masker on the right thigh, Site I as shown in Fig. 2. the test), might account for the drop in mean TE from 9.5 to $3.7 \mathrm{~dB}$ with masker-test longitudinal separation from 0 to $75 \mathrm{~cm}$ (Fig. 3).

If TE with longitudinal separation of masker and test site on the body can be accounted for simply in terms of differential signalling delays due to differing neural distances, it would follow that the peak in $\mathrm{TE}$ as a function of time intervals between a masker and test site longitudinally separated should: (a) be equal in elevation to the peak of a function obtained when the masker and test are not separated, and (b) show a compensation for the differential signalling delay by a time-interval change from zero to a time interval corresponding to the longitudinal separation and neural signalling speed.

Two conditions were tested. In one case, the test site was on the left thigh, while the masker was on the upper left arm, Locus A in Fig. 2. Locus A provided one of the largest longitudinal separations from the test site, and furthermore had produced the least average amount of TE of any body site tested. The second case was the reverse of the first, viz., the test was on Locus A and the masker was on the left thigh test site. If a change in the peak TE from the simultaneous masker and test onsets occurred in case one, then it should follow that the second case, the reverse, would produce a peak TE with an inversion of precedence.

Ten time intervals from $-20.0 \mathrm{msec}$ to $+20.0 \mathrm{msec}$ were employed for both conditions. When the test site was on the upper arm, $\mathrm{O}$ rested his arm on a high stack of foam rubber cushions in order to provide a horizontal surface for gluing the test vibrator.

The results for each $O$ and the mean are shown in Fig. 5. It would appear that the differential signal delay hypothesis for the body data of Experiment 1 has been supported. In the first place, the mean amount of TE at zero time interval, $3.5 \mathrm{~dB}$ in Fig. 5(a), increases quite abruptly to a peak mean of $8.0 \mathrm{~dB}$ at $-10.0 \mathrm{msec}$, just short of the peak mean threshold elevation of $10 \mathrm{~dB}$ for the body data shown in Fig. 3. Likewise, Fig. 5(b) shows a similar increase from $3.3 \mathrm{~dB}$ at zero time interval to $7.3 \mathrm{~dB}$ at $+10.0 \mathrm{msec}$.

These findings, taken in conjunction with those of the previous experiment, show that, despite longitudinal separations and even lateral separations between masker and test sites, the amount of TE may be raised or lowered to be roughly in accord with the data of Experiment 1 by merely adjusting the appropriate time interval. These time-interval adjustments correspond in direction and range with predictions based on generally accepted neural signalling speeds. It appears from the preceding that the only effect spatial factors have is to change arrival times at some central site or sites. If arrival time is compensated for, the central masking effect is independent of loci stimulated on the trunk.

\section{EXPERIMENT 3}

The foregoing results contrast with those of other masking studies (Rosner, 1961; Schmid, 1961; Sherrick, 1964; Uttal, 1960). These studies have shown a diminution in the amount of masking with small spatial separations between the masker and test and with changes in masker placement from ipsilateral ones to those contralateral to the test site. One major difference between these studies and the present one is the location of the sites of stimulation. The cited investigators employed the fingers as loci, whereas in the present investigation only loci on the trunk have been stimulated. The question arises whether the discrepancies between these previous studies and the present one would continue if the fingers were now employed as sites for stimulation. Furthermore, data on the fingers, taken in conjunction with those of Experiment 1, should provide a comparison for the degree of interaction within the body parts. 


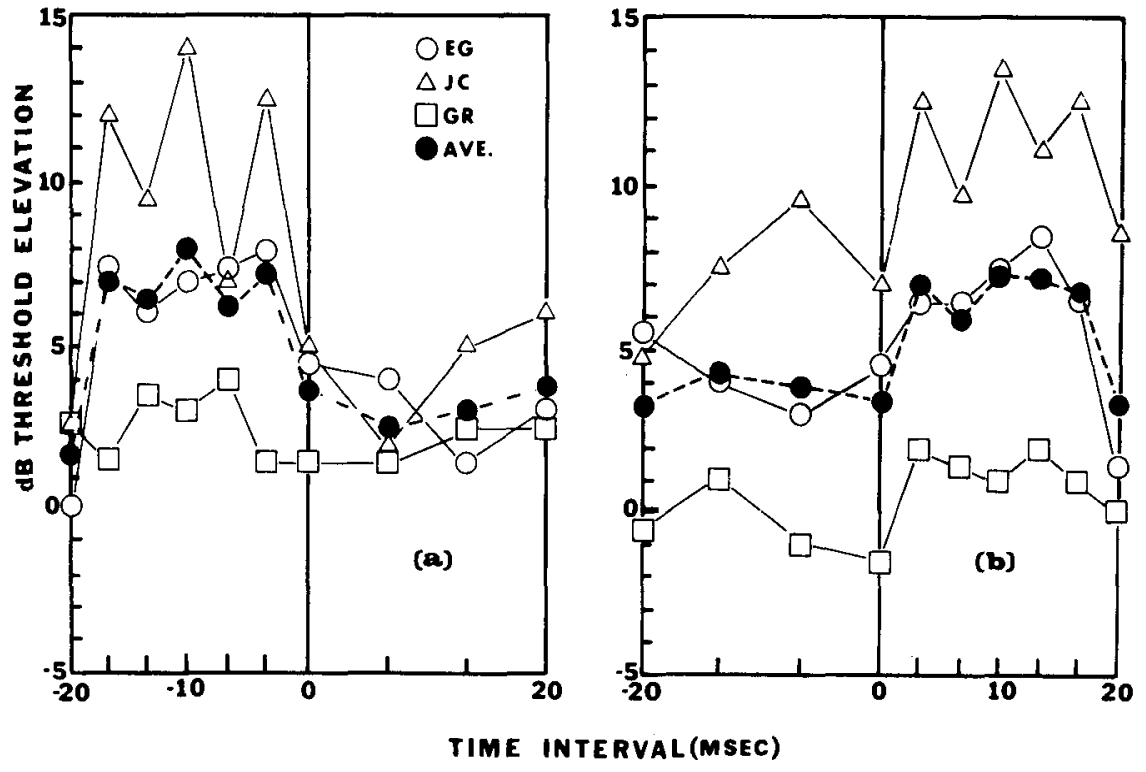

Fig. 5. Threshold elevation as a function of the time interval between masker and test signal onsets. (a) Test site on the lower left thigh; masker on the upper left arm, Site $A$ as shown in Fig. 2. (b) Test site on the upper left arm, Site $A$ as shown in Fig. 2; masker on the lower left thigh.

\section{Apparatus and Procedure}

The Sherrick vibrator previously used in Experiment 1 as a test stimulator was replaced by a Goodmans V-47 vibrator. The latter was fitted with a Lucite ring $(2.2 \mathrm{~cm}$ inside diam, $1.6 \mathrm{~cm}$ long) mounted directly on top of the vibrator's piston. The maskers were Sherrick vibrators modified to stimulate and support the fingers (Gilson, 1968). The skin contactors for the maskers were again Lucite rings with the same dimensions as the test ring. In order to accommodate the Goodmans vibrator, with its lower impedance, the transformer employed in Experiment 1 for the test signal was replaced by a UTC LS-33 that dropped the impedance from 500 to $2.5 \mathrm{ohms}$. With the exception of these changes, the apparatus remained the same as in Experiment 1.

Threshold elevations were determined at the test site, the left index finger, when a masker was placed on each of the other fingers, singly.

\section{Results and Discussion}

Fig. 6 depicts the results for the individual Os as well as the means for the three Os. It appears that, while there are no systematic differences between fingers within the hands, there is a sizeable difference between the hands. A comparison reveals that placement of the masker on nontest fingers of the left hand amounts, on the average, to about twice as much TE as that produced by placement of the maskers on the fingers of the right hand. This difference was significant at the $p<.01$ level with the Wilcoxon Rank Sum test.

The failure to find systematic differences between fingers on one hand contrasts with masking studies employing electrocutaneous stimulation at adjacent and nonadjacent fingers to the test digit (Rosner, 1961; Schmid, 1961; Uttal, 1960). These studies show that the amount of TE decreases with increased masker separation from the test finger by one or more digits.

The difference in stimulus character may account for the apparently conflicting results. In the present case broad contactors producing extensive vibratory wave propagation stimulated large areas of the hand with relatively long bursts of repetitive mechanical impacts, whereas the cited investigators employed sharply localized, single pulses of electrocutaneous stimulation.

The two-fold decrease in TE resulting from moving the masker from the fingers of the test hand to the fingers on the contralateral hand is in close agreement with Sherrick's (1964) findings. This difference, however, is at variance with the results of Experiment 1, that show no significant difference between contralateral and ipsilateral placement of maskers with respect to the thigh test site, when longitudinal separations are similar. The reason for these conflicting results between the body and finger data is not readily apparent. Therefore, one further experiment was carried out to resolve some of the discrepancies.

The finger test site, the left index finger, was examined for TE with a masker at either the body test site of Experiment 1 , the left thigh, or at the contralateral-homologous locus on the right thigh. Conversely, the thigh test site was examined, with maskers placed on either the left or right index fingers.

The results show $7.11 \mathrm{~dB}$ and $4.11 \mathrm{~dB}$ of TE for the test site at the left index finger with maskers on the left and right thighs, respectively, and $1.89 \mathrm{~dB}$ and $2.17 \mathrm{~dB}$ of TE for the test site at the left thigh with maskers at the left and right index fingers, respectively. These figures are based on three observations per $\mathrm{O}$ or a total of nine observations for each combination of masker and test site. A comparison of results shows that, with the finger test site, ipsilateral-contralateral masker placement on the thighs produced a significant difference $(p<.01)$ in TE. Moreover, the obtained values are

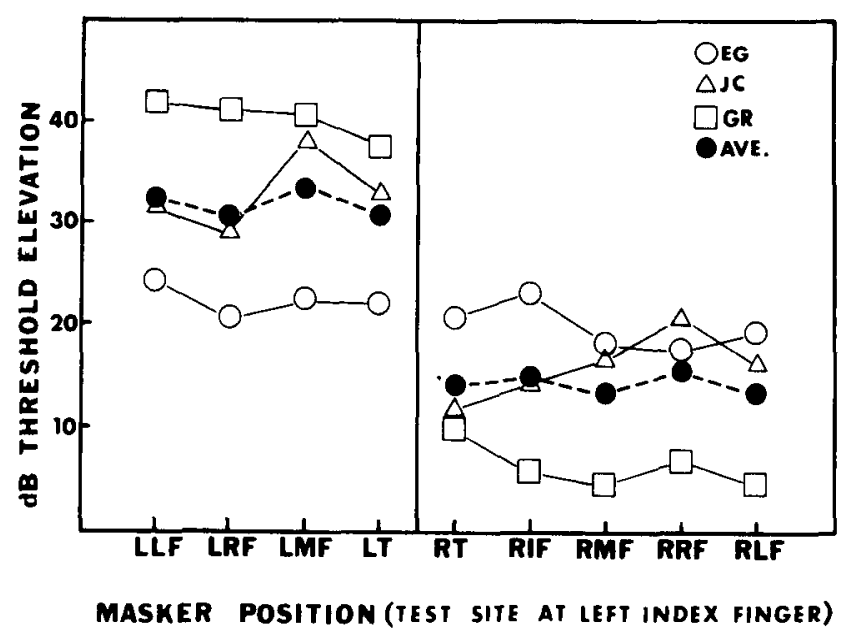

Fig. 6. Threshold elevation at the left index fingertip (LIF) test site, as a function of a masker placed on each of the other fingers, singly. 
roughly of the same $2: 1$ ratio of magnitude as was previously found for the finger test site with ipsilateral and contralateral finger maskers. This comparison accompanies the finding that with the test site on the thigh, masker placement on either the left or right index fingers resulted in no significant difference in TE $(p>.10)$. The latter duplicates the finding for the test site on the thigh and masker placement at body loci in Experiment 1. Whatever is causing the difference or lack of a difference between ipsilateral and contralateral masker placement is associated with the test site because the effect is dependent on the location of the test site (thigh or finger) and not on the manipulation of masker sites between trunk and finger loci.

One further point may be noted by comparing the results of Experiments 1 and 3 . Threshold elevations at the finger test site were far greater than TE at the thigh test site. It would appear that there is a greater amount of interaction between finger loci than bodily loci. However, there is a question as to the validity of such a comparison, since threshold determinations were made at different test loci. One way of testing this validity is to examine the finger and thigh test sites to determine if both would yield commensurate results under similar conditions. Such a comparison reveals that $7.11 \mathrm{~dB}$ of $\mathrm{TE}$ with the left index finger as the test site and the left thigh as the masker locus clearly does not equal the $1.89 \mathrm{~dB}$ of TE obtained with the left thigh as the test site and the left index finger as the masker locus, even though the same two loci were used in both cases $(\mathrm{p}<.01$, by the Wilcoxon Signed Rank test).

In spite of these large differences in $\mathrm{TE}$ it was possible that the two test sites may have different loudness growth functions with intensity and might be equatable indirectly if this function were known. A pilot experiment, undertaken to assess the relative loudness growths at the two test sites, however, showed only a small dissimilarity between the finger and thigh, a dissimilarity insufficient to explain the large differences in TE observed. Thus, comparison between the finger results of Experiment 3 and the body results of Experiment 1 is inappropriate, and comparisons should be limited to those data obtained with the same test site.

Experiment 3 clearly indicates that there are limitations to the rather simple findings of Experiments 1 and 2, which show that degree of vibrotactile masking is controlled by differential time delays, and not by masker placement. Furthermore, the differences in degree of contralateral masking for the finger and thigh test sites give evidence for some fundamental difference in the neural organization of the body, compared to the fingers, that is more subtle than simply the amount of sensory cortex subserving the loci in question.

\section{REFERENCES}

ALLUISI, E. A., MORGAN, B., \& HAWKES, G. Masking of cutaneous sensations in multiple stimulus presentations. Perceptual \& Motor Sk ills, 1965, 20, 3945.

BÉKÉSY, G. V. Human skin perception of traveling waves similar to those on the cochlea. Journal of the Acoustical Society of America, $1955,27,155-161$.

BENDER, M. B. Disorders in perception. Springfield, Ill.: Thomas, 1952.

CRAIG, J. C. Vibrotactile loudness addition. Perception \& Psychophysics, 1966, 1, 185-190.

GELDARD, F. A. Cutaneous coding of optical signals: The optohapt. Perception \& Psychophysics, 1966, 1, 377-381.

GELDARD, F. A., \& SHERRICK, C. E. Multiple cutaneous stimulation: The discrimination of vibratory patterns. Journal of the Acoustical Society of America, 1965, 37, 797-801.

GILSON, R. D. Some factors affecting the spatial discrimination of vibrotactile patterns. Perception \& Psychophysics, 1968, 3, 131-136.

GOLLA, F., \& ANTONOVITCH, S. The rate of conduction in the human motor nerve. Brain, 1931, 54, 492-507.

HALLIDAY, A. M., \& MINGAY, R. Retroactive raising of a sensory threshold by a contralateral stimulus. Quarterly Journal of Experimental Psychology, 1961, 13, 1-11.

MAYER, R. F. Nerve conduction studies in man. Neurology, 1963, 13, $1021-1030$

ROSNER, B. S. Neural factors limiting cutaneous spatiotemporal discriminations. In W. A. Rosenblith (Ed.), Sensory communication. New York: Wiley, 1961.

SCHMID, E. Temporal aspects of cutaneous interaction with two-point electrical stimulation. Journal of Experimental Psychology, 1961, 61, 400-409.

SHERRICK, C. E. Observations relating to some common psychophysical functions as applied to the skin. In G. R. Hawkes (Ed.), Symposium on cutaneous sensitivity. U. S. Army Medical Research Laboratory, Ft. Knox, Ky., 1960.

SHERRICK, C. E. Effects of double simultaneous stimulation of the skin. American Journal of Psychology, 1964, 77, 42-53.

SHERRICK, C. E. Simple electromechanical vibration transducer. Review of Scientific Instruments, 1965, 36, 1893-1894.

UTTAL, W. R. Inhibitory interaction of responses to electrical stimuli in the fingers. Journal of Comparative \& Physiological Psychology, 1960, $53,47-51$.

YAI, $H$. The effects of side-pressure on pressure sensation. Japanese Journal of Psychology, 1959, 29, 303-312.

\section{NOTES}

1. This study was supported by Grant NB-04755 from the National Institutes of Health, U. S. Department of Health, Education and Welfare. 2. Now at Naval Aerospace Medical Institute, Pensacola, Fla. 32512.

(Accepted for publication September 20, 1968.) 